\title{
THE EFFECTS OF MICROSTIMULATION AND MICROLESIONS IN THE VENTRAL AND DORSAL RESPIRATORY GROUPS IN MEDULLA OF CAT T $^{1}$
}

\author{
DEXTER F. SPECK ${ }^{2}$ AND JACK L. FELDMAN \\ Departments of Physiology and Anesthesia, Northwestern University, Chicago, Illinois 60611
}

Received October 2, 1981; Revised January 4, 1982; Accepted January 12, 1982

\begin{abstract}
The responses in respiratory outflow resulting from microstimulation and successive microlesions of the dorsal (DRG) and ventral (VRG) respiratory groups of neurons in the brainstem were studied in anesthetized, paralyzed, artificially ventilated cats. Microstimulation (2 to $120 \mathrm{~Hz} ; 5$ to $50 \mu \mathrm{A} ; 100$ $\mu$ sec pulse duration) at almost every point within the DRG or VRG produced a bilateral short latency inhibition of phrenic nerve activity which had an onset latency of 4 to 9 msec and a duration of 4 to 25 msec. This global stereotyped phrenic inhibition was elicited by single pulses and often was accompanied by a postinhibitory excitation. In $48 \%\left({ }^{92} / 193\right)$ of the stimulation trials, trains of stimulus pulses during inspiration decreased the duration of inspiration. In $25 \%$ of the expiratory microstimulation trials, expiratory duration was increased and in $11 \%$, expiration was shortened markedly by trains of pulses. Single shocks delivered to the right VRG or DRG produced a short latency excitation in the ipsilateral recurrent laryngeal nerve (RRL). This RRL excitation had an onset latency of 2 to $5 \mathrm{msec}$ and a duration of 3 to $15 \mathrm{msec}$. Evidence suggests that the RRL excitation is due to a paucisynaptic activation of expiratory motoneurons in the caudal VRG. This activation is synchronous with the inhibition of inspiratory neurons in DRG and VRG.

Despite the powerful short latency effects of microstimulation in VRG and DRG, extensive bilateral destruction of these neuronal populations had only modest effects on respiratory rhythm, while it decreased (or abolished) respiratory outflow in phrenic and recurrent laryngeal nerves. The combined results of the microstimulation and microlesion portions of this study suggest that a region (or regions) outside of the DRG and VRG might be important in the control of the respiratory pattern and that the DRG and VRG are important in determining the depth of inspiration; their role in generating respiratory rhythm needs to be critically re-examined.
\end{abstract}

Mammalian respiratory rhythm probably is generated within the brainstem (Binet et al., 1953; Hoff and Breckenridge, 1949; Hukahara, 1976; Pitts et al., 1939; St. John et al., 1981; Wang et al., 1957; see, though, Aoki et al., 1980). Neurons with respiration-modulated firing patterns are found throughout the pons and medulla (Vibert

\footnotetext{
${ }^{1}$ This work was supported by National Institutes of Health Grants HL-23820, HL-00554, and HL-06331 and National Institute of Mental Health Grant T32-MH16097. Preliminary results have been presented as abstracts (Speck, D. F., and J. L. Feldman (1981) Fed. Proc. 40: 429; Speck, D. F., and J. L. Feldman (1981) Soc. Neurosci. Abstr. 7: 941). We would like to thank $\mathrm{H}$. Jakiela for providing assistance in computer software and hardware designs and G. L. Schaps for his excellent technical assistance. Morton I. Cohen, Alan Gibson, and Peter Kirkwood provided valuable comments on the initial drafts of this paper.

${ }^{2}$ To whom correspondence should be addressed at Department of Physiology, Northwestern University, 303 E. Chicago Avenue, Chicago, IL 60611.
}

et al., 1976). However, respiration-related neurons are most densely concentrated in two anatomically distinct locales, the dorsal respiratory group (DRG) in the region of the ventrolateral nucleus of tractus solitarius (vlNTS) which lies 0 to $2.5 \mathrm{~mm}$ rostral to the obex and the ventral respiratory group (VRG) in the region of the nucleus retroambigualis (NRA) which extends from the spinomedullary junction rostral to the retrofacial nucleus, including the so-called "Botzinger Complex" (Merrill, 1981). An underlying assumption of most current research on brainstem mechanisms for respiratory rhythmogenesis is that the rhythm is generated by a subset of propriobulbar neurons concentrated in the VRG and/or DRG (see reviews by Cohen, 1979; Mitchell and Berger, 1981; Wyman, 1977). However, the role of neurons in these locales in respiratory rhythmogenesis and/or pattern generation is obscure. Unit recording experiments have led to the identification of the spontaneous trajec- 
tories of respiratory neuronal membrane potentials (e.g., Richter et al., 1975) and their response properties to a variety of stimuli, such as lung inflation (e.g., Feldman et al., 1976), $\mathrm{CO}_{2}$ (e.g., Cohen, 1968; St. John and Wang, 1977b), and laryngeal nerve stimulation (e.g., Berger, 1977). Anatomical tracing (Bystrzycka, 1980; Kalia et al., 1979) and antidromic mapping experiments (Bianchi, 1971; Merrill, 1974) have shown a diverse set of both propriobulbar and spinobulbar projections. Except for those neurons shown either to project directly to spinal cord respiratory motoneurons (e.g., phrenic and intercostal) and therefore act as upper motoneurons (Merrill, 1974 ) or to be laryngeal-pharyngeal respiratory motoneurons, the functional role of any given neuron type or, in fact, any region as a whole is unknown.

Previous investigators have tried to determine the role of the DRG in the central generation of the respiratory pattern by studying the effects of lesions in the DRG on respiratory nerve or muscle activity. Oberholzer and Tofani (1965) reported that bilateral destruction of the DRG in anesthetized, vagotomized rabbits caused only slight changes in the respiratory pattern. Similarly, Berger and Cooney (1981) observed that extensive destruction of vINTS neurons with kainic acid had little effect on respiratory rhythm in the conscious cat. In contrast, Koepchen et al. (1974) and Brodie and Borison (1957) reported that vINTS lesions produced apneusis. An important conclusion from these latter two experiments is that vlNTS lesions did not alter the generation of the augmenting "ramp" of inspiratory discharge significantly, although they might alter the mechanisms for inspiratory timing.

The importance of the VRG in rhythm generation is suggested by the observation that transections or lesions in the medulla, rostral to the DRG but including the rostral VRG, may reduce apneusis (St. John and Wang, 1977a) or produce gasping (Wang et al., 1957). Gromysz et al. (1980) reported that respiratory patterns in the rabbit were not affected by reversible procaine lesions within the caudal NRA, nor did unilateral transections $4.0 \mathrm{~mm}$ rostral to the obex alter respiratory rhythmicity greatly (Gromysz and Karczewski, 1980; Karczewski and Gromysz, 1980). However, similar lesions in the rostral NRA, the retrofacial nucleus, and the facial nucleus did alter respiratory rhythm in a consistent fashion.

The present experiments were designed to investigate further the possible roles of the VRG and DRG neurons in the generation of respiratory rhythm. Most current information concerning these medullary nuclei is based on data obtained with extracellular recording techniques. These methods have very serious limitations, such as the inability to isolate and record from small spiking and/or nonspiking neurons. Since both of these types of neurons may play a role in respiratory rhythmogenesis, extracellular recording techniques may be inappropriate to locate the respiratory oscillator; these neurons would, however, respond to local microstimulation and microlesion. Therefore, both microstimulation ( 5 to $50 \mu \mathrm{A} ; 1$ to 120 $\mathrm{Hz}$; Asanuma, 1977) and successive microlesions (focal destruction of each lesion $<700 \mu \mathrm{m}$ in diameter) were performed in these medullary respiratory regions, while the responses in respiratory outflow from the phrenic
(Phr) nerves and the right recurrent laryngeal nerve (RRL) were monitored closely.

\section{Materials and Methods}

Experiments were performed on 18 adult cats (1.5 to $5.0 \mathrm{~kg}$ ) anesthetized with chloralose/urethane (40 and $250 \mathrm{mg} / \mathrm{kg}$, i.v., respectively). Dexamethasone $(2 \mathrm{mg}$, i.m.) was given at the start of each experiment to minimize the swelling of the brainstem and cerebellum. Subsequent to tracheotomy and cannulation of the femoral artery and vein, cats were placed in a rigid head holder, paralyzed with gallamine triethiodide, thoracotomized, and artificially ventilated with $100 \%$ oxygen. In 7 experiments involving recurrent laryngeal nerve recording, the vagi were left intact; otherwise bilateral cervical vagotomies were performed. Cats with intact vagi were ventilated with a cycle-triggered pump (Feldman et al., 1976) that inflated their lungs coincident with phrenic discharge; this permits the respiratory period to be determined centrally. The end tidal percentage of $\mathrm{CO}_{2}$ was regulated at $5 \pm 0.5 \%$ and body temperature was maintained at $38 \pm 0.5^{\circ} \mathrm{C}$.

Systemic arterial pressure was monitored from the femoral arterial catheter with a pressure transducer. An occipital craniotomy was performed and the dura was cut to expose the cerebellum and brainstem. The C5 phrenic roots were isolated via a dorsal approach, cut, and desheathed. The right recurrent laryngeal nerve was dissected dorsal to the trachea, cut, tied, and pulled over the muscle into the subscapular space. Nerves were placed across bipolar silver wire electrodes connected to high impedance differential instrumentation amplifiers. Phrenic nerve activity was fed into a "phrenic processor" that derived a gate marking the period of phrenic nerve activity (neural inspiration) and phrenic nerve silence (neural expiration). In most experiments, one or two tungsten microelectrodes (tip size $=10 \mu \mathrm{m}$; resistance $=$ 1.5 megohms, $1 \mathrm{kHz}$ ) suitable for recording, microstimulation, and microlesioning were inserted into the regions of the inspiration-modulated neuronal activity. These regions were located in either the VRG from $1.0 \mathrm{~mm}$ caudal to $5.0 \mathrm{~mm}$ rostral to the obex and/or the DRG from 0.0 to $2.5 \mathrm{~mm}$ rostral to the obex; the most rostral portions of the VRG had both inspiratory and expiratory activity (Merrill, 1970). The electrodes were connected to a manual switch that led either to: $(a)$ a high impedance source follower and subsequent second stage amplification and filtering, $(b)$ a constant current stimulator, or (c) a DC current lesion maker. Thus, we could record, microstimulate, and subsequently lesion from the same electrode with relative ease. In a total of 13 experiments, stimulus pulses ( 80 to $100 \mu \mathrm{sec}$ ) of various frequencies (1 to $120 \mathrm{~Hz}$ ) and intensities (5 to $30 \mu \mathrm{A}$ typical, $50 \mu \mathrm{A}$ maximal) were delivered through the tungsten microelectrodes. Stimulus trains were either continuous or gated with variable delays from the onset or offset of phrenic nerve activity. Stimulus intensities were monitored by measuring the voltage drop across a $100-\mathrm{ohm}$ resistor in the ground path of the current loop.

All recording sessions started with insertion of one or more microelectrodes in the right VRG. At any given microelectrode position in the VRG, the spontaneous 
activity on the microelectrodes and nerves was recorded on analog tape. Then the responses of the nerves and of the units on a second electrode (if there was one in a region of interest) to a variety of stimulus variables were recorded. Subsequent to this procedure, if the electrode tip was in the middle of the dorsoventral extent of respiration-modulated units in a given penetration, a small lesion was made by passing 10 to $20 \mu \mathrm{A}$ of DC cathodal current through the electrode tip for 20 to 40 sec. Such lesions were 250 to $500 \mu \mathrm{m}$ in diameter (see Fig. 1 , cat 300 ). Within 5 min after the lesion, a recording of spontaneous activity at the lesion site was made. Usually no unit activity was detected within 250 to $350 \mu \mathrm{m}$ of the center of the lesion. If respiration-modulated activity was observed on the same puncture within $1000 \mu \mathrm{m}$ of the recording/lesion site, the stimulation-lesion protocol was repeated; otherwise, the electrode was removed and reinserted within $500 \mu \mathrm{m}$ of the previous puncture. This procedure was repeated until we had lesioned the right VRG extensively and could find no further respirationmodulated activity rostral and no inspiratory activity caudal. The electrode then was repositioned in the right DRG and the above procedure was iterated. In this manner, microstimulation tests were performed at 209 distinct anatomical sites located along 106 electrode tracks in the right DRG and VRG of 13 cats. In 6 experiments, after extensively lesioning two populations, we continued to lesion the contralateral populations, skipping the determination of the responses to microstimulation.

In 4 experiments, arrays of electrodes were used to maximize lesion size within a given population and the speed at which extensive lesions could be made. These electrode arrays were made by epoxying four insulated tungsten microelectrodes together in a row so that the tips were $1.0 \mathrm{~mm}$ apart. In most cases, three or four of the microelectrodes in a rostrocaudally oriented array could be positioned successfully within the same respiration-modulated neuronal population. After recording control unit and nerve activities, successive lesions (20 $\mu \mathrm{A} ; 40 \mathrm{sec}$; electrode negative) were made on each electrode that demonstrated respiration-modulated activity. Lesions then were made between pairs of adjacent electrodes with current flowing in one direction for $30 \mathrm{sec}$ and then reversed for an additional $30 \mathrm{sec}$. Histology verified that such lesions were rostrocaudally oriented cylinders approximately 400 to $900 \mu \mathrm{m}$ in diameter and extending for distances of approximately $1 \mathrm{~mm}$ (Fig. 1, cat 345).

Data analysis. The effects of brainstem microstimulation were analyzed for both short latency effects and for changes in the overall respiratory pattern. To determine changes in respiratory outflow from the phrenic and recurrent laryngeal nerves, the recorded data were played back and displayed on a storage oscilloscope. Gross changes ( $>10 \%$ of control values) in nerve signal amplitudes and the duration of inspiration and expiration elicited by microstimulation were readily observed on slow sweep speed traces. The data also were displayed at faster sweep speeds with the oscilloscope triggered by the recorded stimulus pulses. Whenever a short latency response 10 stimulation was observed in a single trial in any of the recorded signals, the response was examined more closely by using a laboratory satellite computer to determine the latency and duration of the response to individual stimulus pulses. Analog signals were converted to 12 -bit digital records by sampling at 10,000 to 15,000 samples/sec after low pass filtering at $5 \mathrm{kHz}$ to prevent aliasing. Digital records were displayed on a graphics monitor; the $x$ and $y$ axes could be shifted or scaled as desired. Movable timing cursors were superimposed on the records to measure response latencies. Peristimulus time histograms also could be computed (Fig. 2). Thus, accurate high resolution measurements could be obtained from the short latency responses to brainstem stimulation. Finally, these displays could be converted to hard copy printouts seen in Figures 2, 3, and 6.

To examine the effects of multiple microlesions, recordings of spontaneous respiration were obtained before and after each lesion. Representative segments of the integrated phrenic nerve and/or recurrent laryngeal nerve signals (time constant $=50 \mathrm{msec}$ ) were sampled at 500 samples/sec and were converted to 12-bit digital records and stored to be used for two purposes. First, the data traces were displayed in three-dimensional chronological plots (Figs. 7 and 8) which demonstrated any gross changes in nerve amplitude and timing that occurred after (and between) each experimental lesion. Secondly, the individual records were computer analyzed to determine the duration of inspiration and expiration and the end inspiratory amplitude of phrenic activity. Data were obtained from 10 consecutive respiratory cycles and the mean and standard deviation were calculated. These data then could be displayed as seen in Figures 10 and 11.

\section{Results}

Effects of stimulation. In 7 experiments, the vagus nerves were left intact and right recurrent laryngeal nerve activity (RRL) was recorded. Microstimulation of either the right. DRG or the right VRG with either single shocks or trains of pulses elicited a short latency excitation of the ipsilateral RRL. The latency of this response ranged from 2 to $5 \mathrm{msec}$. The duration of the excitation ranged from 3 to $15 \mathrm{msec}$ (Fig. $2 B$ ). Occasionally, a postexcitatory inhibition which had a duration of 5 to 15 msec was observed. At many stimulation sites, the RRL response to microstimulation was dependent upon the

Figure 1. Histological verification of lesion sites in the DRG and VRG; these are labeled in the figure by the associated anatomical nuclei (vINTS and NRA). Transverse cut Nissl-stained sections are approximately $1.0 \mathrm{~mm}$ rostral to the obex. Lesions in cat 300 were made by passing $15 \mu \mathrm{A}$, electrode negative, for $30 \mathrm{sec}$. This lesion protocol consistently produced a lesioned area of 250 to $500 \mu \mathrm{m}$ in diameter. Lesions are seen in the right vINTS and NRA. Neuronal destruction is present in the left vlNTS and NRA but is not visible at this magnification. Lesions in cat 345 were made by passing $20 \mu \mathrm{A}$ between adjacent electrodes in an array. Current was passed for $30 \mathrm{sec}$ in one direction and then the polarity was reversed for an additional 30 sec. These lesions were 300 to $600 \mu \mathrm{m}$ in diameter and extended for distances greater than $1 \mathrm{~mm}$. Lesions are apparent in the left and right vlNTS and NRA. 


\section{CAT 300}

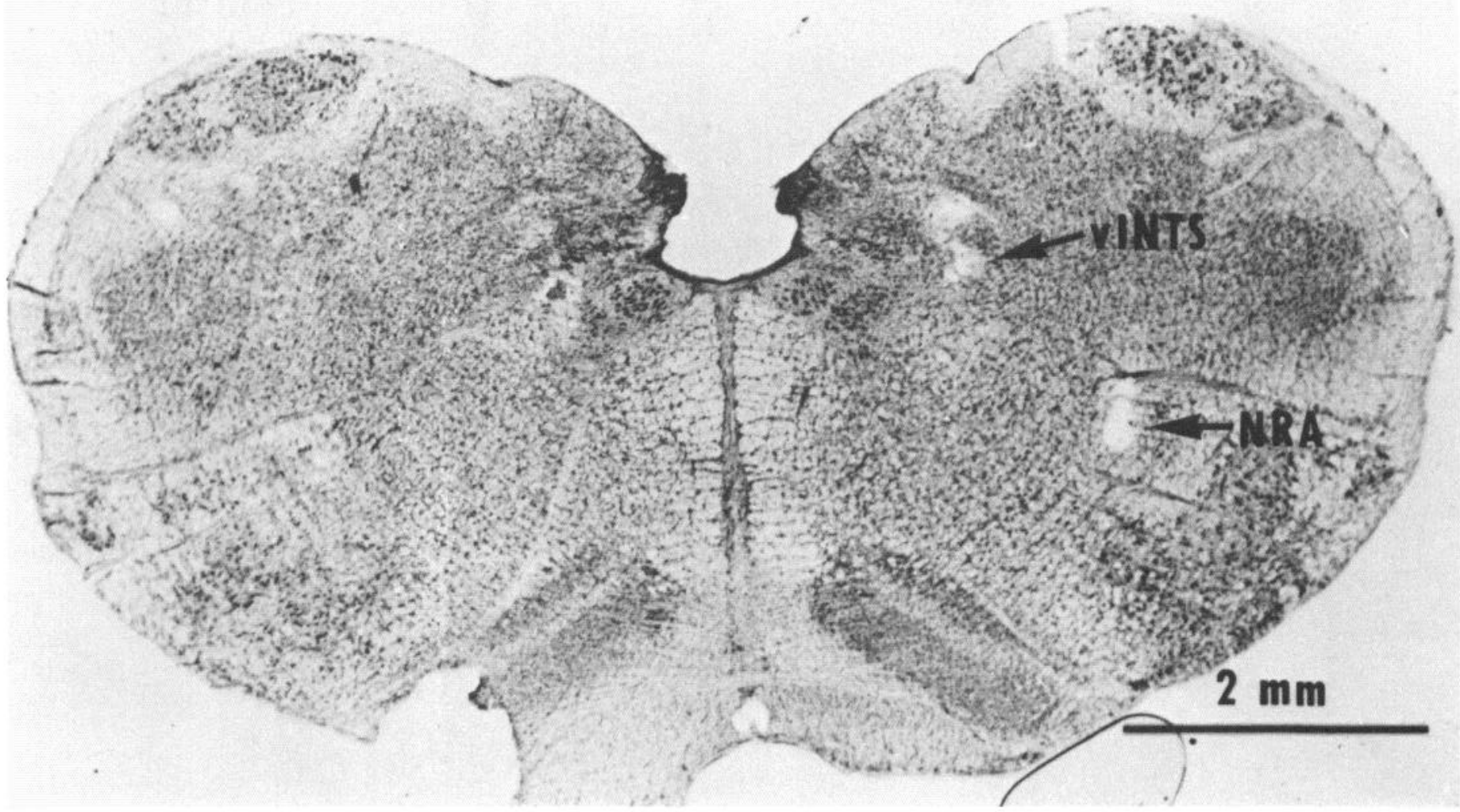

\section{CAT 345}

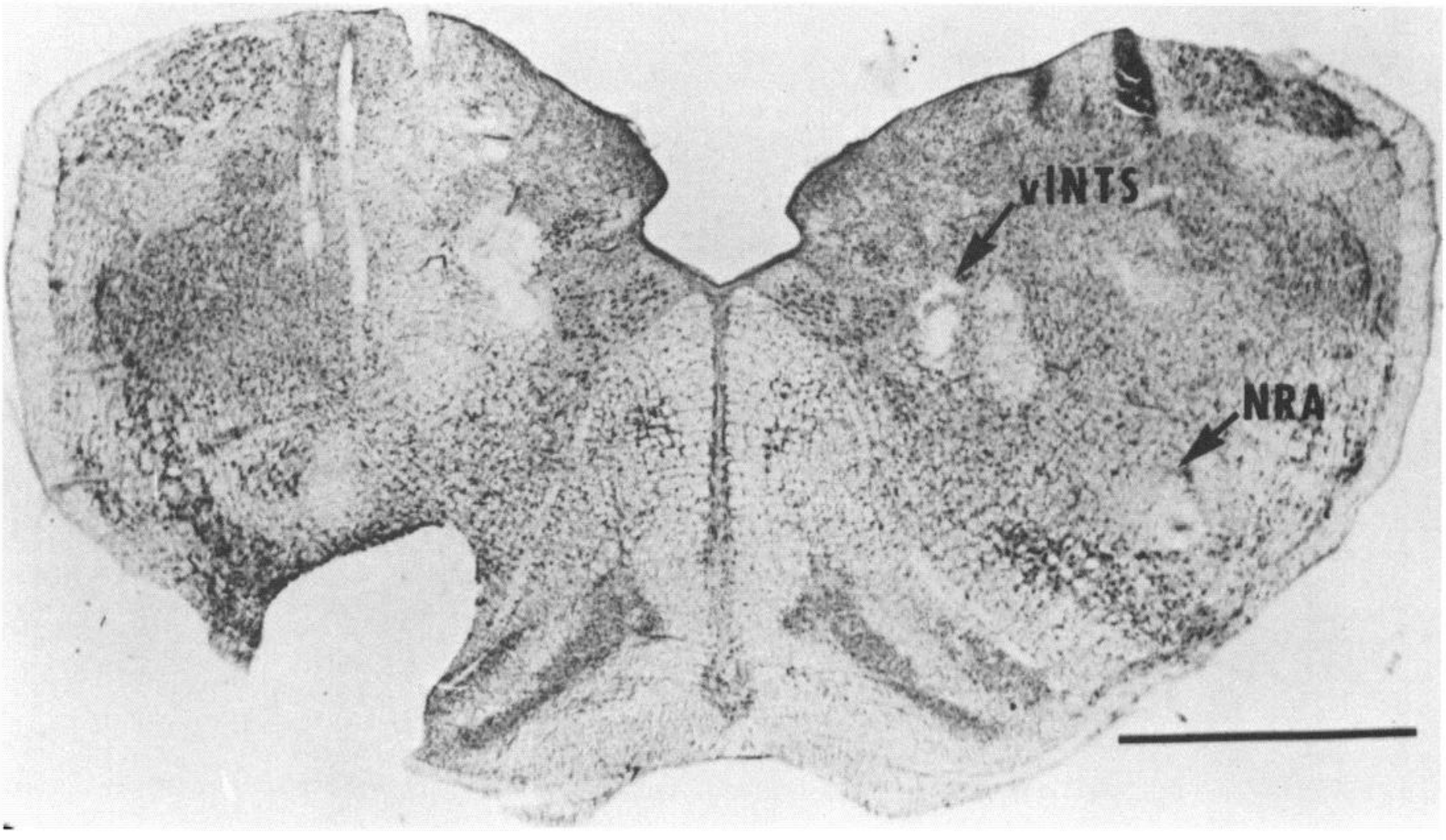


A
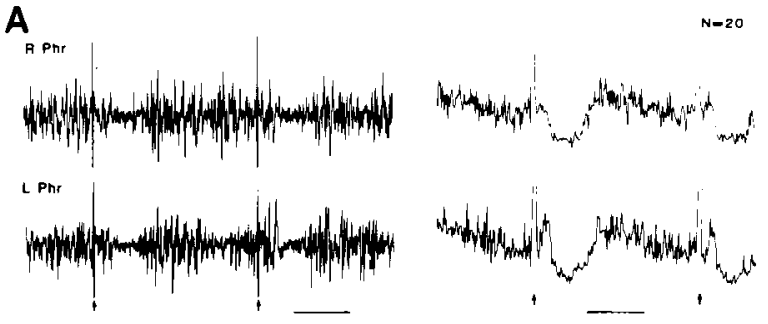

B

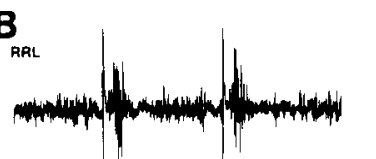

L Pnr
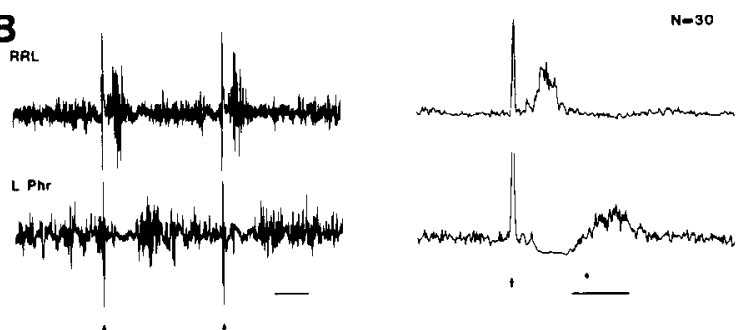

C

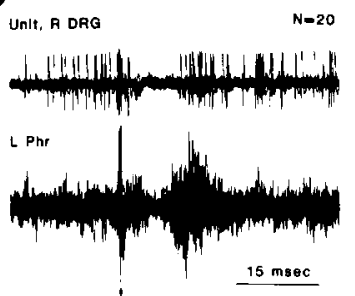

Figure 2. Short latency responses to microstimulation of the right VRG approximately $1.0 \mathrm{~mm}$ rostral to the obex. The left panels demonstrate typical responses observed in simultaneous recordings of: $(A)$ the right phrenic $(R P h r)$ and left phrenic $(L P h r)$ nerve, $(B)$ the $\mathrm{L} \mathrm{Phr}$ and right recurrent laryngeal nerve ( $R R L)$, and $(C)$ the $\mathrm{L}$ Phr and single units in the vlNTS. Stimulus pulses are marked by arrows. Twenty traces have been superimposed in $C$ to clearly show the inhibition that microstimulation elicits in single units. The right panels in $A$ and $B$ show poststimulus histograms constructed from the same experimental runs as the records in the left panels; 20 to 30 traces from two inspiratory cycles were used to construct these histograms. Note the bilateral short latency phrenic inhibition and the postinhibitory excitation elicited by each stimulus pulse in $A, B$, and $C$. In $A$, a short latency excitation which precedes the inhibition is seen in the contralateral phrenic ( $L P h r$ ) nerve. This response was observed sometimes. Also note the RRL excitation that occurs with approximately the same latency as the phrenic inhibition. Similar responses were seen in all experiments when stimulating at any point in the DRG or VRG. The solid bar indicates $15 \mathrm{msec}$ in all panels.

phase of respiration. In such cases, RRL excitation was more pronounced during expiration. After extensive lesioning of the rostral right VRG, RRL activity usually lost its respiration-modulated pattern. However, microstimulation in right VRG at unlesioned sites still continued to elicit a normal excitation of the RRL with excitation more pronounced during expiration. Stimulation of the RRL often evoked antidromic unitary spikes or field potentials from microelectrodes in the right VRG at a latency of 2 to $3 \mathrm{msec}$.

Single shocks of microstimulation (10 to $35 \mu \mathrm{A} ; 100$ $\mu$ sec duration) in either the DRG or the VRG elicited a short latency "inhibition" of phrenic nerve activity (Fig. $2)$. (This inhibition may be due to: $(a)$ pre- or postsynaptic inhibition of phrenic motoneurons and/or $(b)$ dis- facilitation of phrenic motoneurons.) When microstimulation was applied to the right VRG, transient inhibition was observed in both the left and the right phrenic nerve activity (Fig. $2 A$ ). Similarly, in 12 of 14 cases, inspiratory unit activity recorded from a second microelectrode positioned in either the ipsilateral VRG or DRG was inhibited by VRG stimulation (Fig. 2C). (Once again, we did not determine if this response is due to a synaptic inhibition of these inspiratory neurons or if it is the result of a disfacilitation.) In two cases, activity in VRG units was elicited at short latency by ipsilateral VRG microstimulation. We were unable to determine if these unitary responses were due to recruitment of a neuron normally silent during inspiration or to activation of an inspirationmodulated neuron that was below threshold.

Transient short latency phrenic inhibition could be initiated by single shocks of microstimulation at almost every site where respiration-modulated activity could be recorded $(92 \%(193 / 209)$ of the stimulation trials produced a short latency response); however, when microstimulation at similar parameters (and even stronger) was applied to adjacent areas lacking respiration-modulated activity, there was no change in phrenic output. The latency between stimulation and the onset of phrenic inhibition ranged from 4 to $9 \mathrm{msec}$, and the duration of inhibition ranged from 4 to $25 \mathrm{msec}$. No correlation was observed between the stimulation sites in the DRG or VRG and the degree of inhibition. Occasionally, a short latency (1.8- to $4.0-\mathrm{msec}$ ) contralateral phrenic nerve excitation preceded the inhibition (Fig. $2 A, L P H R$ ). Quite often, the phrenic inhibition was followed by a short period of postinhibitory excitation which lasted from 8 to $25 \mathrm{msec}$. Increasing stimulation currents and frequencies above the threshold stimulation parameters had a modest effect on the response characteristics and the response quickly saturated. The example in Figure 3 demonstrates that, as the stimulus current was increased from 10 to $30 \mu \mathrm{A}$, the degree of phrenic inhibition became more marked; however, above $30 \mu \mathrm{A}$, there appeared to be little difference in the short latency response to stimulation.

Despite the fact that short latency phrenic inhibition was almost always observed following a single shock (Fig. 2 ), trains of pulses (5 to $25 \mu \mathrm{A} ; 10$ to $50 \mathrm{~Hz}$ ) did not appear to alter the gross pattern of phrenic discharge significantly in approximately $52 \%(101 / 193)$ of the stimulation trials. In such cases, the respiratory frequency, inspiratory duration, and peak phrenic activity did not change. In $48 \%\left({ }^{92 / 193)}\right.$ of these stimulation trials, trains of pulses caused a premature termination of phrenic activity (Fig. 5A). This inspiratory termination varied greatly in its sensitivity, but it could be observed occasionally after as few as three stimulus pulses. A close examination of the short latency responses indicated that, when the stimulation prematurely terminated inspiration, the duration of phrenic inhibition increased until finally phrenic activity was abolished completely (Fig. $4 A$ ). However, if inspiratory duration was unaltered by stimulation, the duration of the short latency phrenic inhibition was lessened as the inspiratory period progressed (Fig. $4 B$ ).

Several different responses to microstimulation during expiration were observed. The most common response 

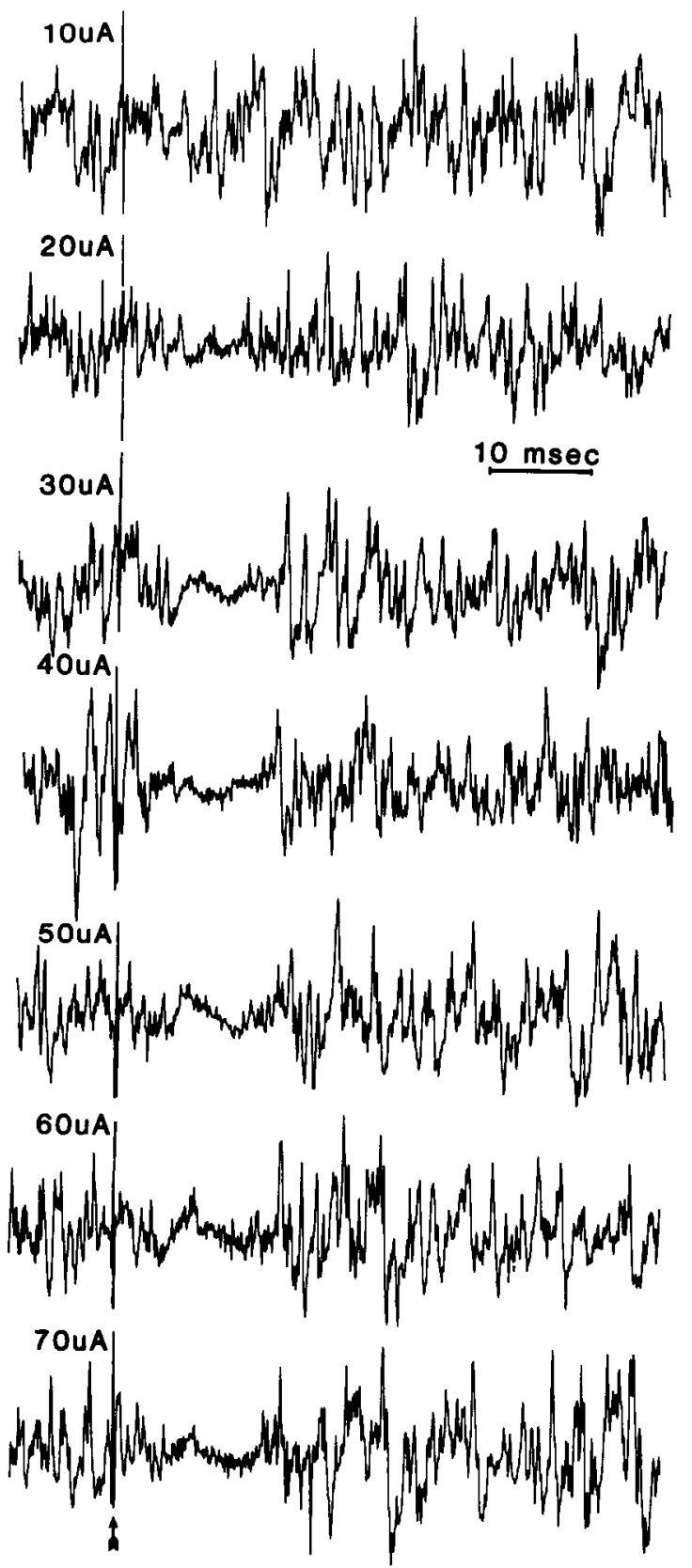

Figure 3. Effects of varying stimulus currents on the left phrenic response to microstimulation of the right VRG. At 10 $\mu \mathrm{A}, 20 \mathrm{~Hz}$, and $100 \mu \mathrm{sec}$ pulse duration, stimulation had little effect on left phrenic discharge. Increasing stimulus current to $20 \mu \mathrm{A}$ produced a weak short latency inhibition; $30 \mu \mathrm{A}$ produced a marked phrenic inhibition. This response quickly saturated and did not change as stimulus currents were increased further. Stimulus pulses are marked by the arrow.

produced by expiratory stimulation ( ${ }^{123 / 193}$ microstimulation trials) was no effect on the duration of that cycle (Fig. $6 A$ ). In 49 out of 193 trials, there was an increase in expiratory duration which was always associated with the termination of inspiration by stimulation starting during inspiration (Fig. 5). This prolongation of expiration usually lasted until the stimulation ceased, at which time there was an immediate onset of inspiratory activity (Fig. 6B). Occasionally, expiratory microstimulation would cause an increase in expiratory duration which persisted for several seconds following cessation of stimulation. In several cases, microstimulation at parameters as low as $10 \mathrm{~Hz}$ and $15 \mu \mathrm{A}$ was sufficient to prevent the onset of inspiratory activity for periods exceeding $20 \mathrm{sec}$. A normal phrenic inspiratory discharge usually followed this type of response (Fig. 6D); however, occasionally, the poststimulation inspiration was reduced markedly in amplitude (Fig. $6 \mathrm{C}$ ). The prolongation of expiration was elicited by microstimulation at numerous points in the DRG or VRG; however, there did not appear to be a spatial pattern associated with the effective stimulation sites. A third type of response to expiratory stimulation involved the shortening of expiration and the subsequent increase in respiratory frequency (Fig. 6E). Twenty-one examples of this response were observed and it is interesting to note that inspiratory stimulation at almost $62 \%$ $(13 / 21)$ of these sites did not alter the duration of inspiration. The decrease in expiratory duration was observed after microstimulation in the vINTS ( 3 trials), the NRA ( 8 trials), and the Botzinger Complex (9 trials). This type of response occasionally tended to develop slowly and, if the stimulation was continued long enough, sometimes resulted in a very rapid pattern of high amplitude phrenic bursts which resembled that seen during panting.

Effects of lesions. Despite the powerful and virtually ubiquitous short latency effects of microstimulation in the respiratory populations, microlesions at the stimulation sites did not appear to have a significant effect on the timing of phrenic nerve discharge. Successive lesions within the VRG and the DRG did not alter respiratory rhythm consistently, although a very small number of transient alterations in rhythm were observed immediately after lesioning. These transient changes were almost always accompanied by large changes in blood pressure with mean arterial pressure sometimes exceeding $200 \mathrm{~mm} \mathrm{Hg}$ or falling below $80 \mathrm{~mm} \mathrm{Hg}$. It is not clear from these experiments whether the transient respiratory alterations were due to cardiorespiratory interactions or if they were a direct result of the destruction of respiration-related structures. However, the frequent observation of spontaneous respiratory rhythm changes accompanied by spontaneous fluctuations in systemic arterial pressure suggest the former possibility. Increases in blood pressure were accompanied by a decrease in respiratory frequency. Of the 18 cats which were lesioned, 3 exhibited a cessation of inspiratory activity (expiratory apnea), while another 3 became severely apneustic. In 2 of the apneic cats, inspiratory activity stopped after the very first lesion in the right VRG; while in the 3rd, apnea occurred after the entire right VRG was lesioned. Apneusis developed in the 3 cats after lesion of at least two respiratory populations. All of these alterations in respiratory rhythm were accompanied by dramatic fluctuations in arterial pressure. During the lesion procedure, mean arterial pressure often exceeded $200 \mathrm{~mm} \mathrm{Hg}$; subsequent to lesion in these 6 cats, the mean arterial pressure decreased to below $70 \mathrm{~mm} \mathrm{Hg}$.

The only consistent result of VRG microlesioning was a reduction in the amplitude of the signals recorded from the contralateral phrenic and ipsilateral recurrent laryngeal nerves. These reductions in amplitude seldom could be discriminated by eye after a single lesion; however, 

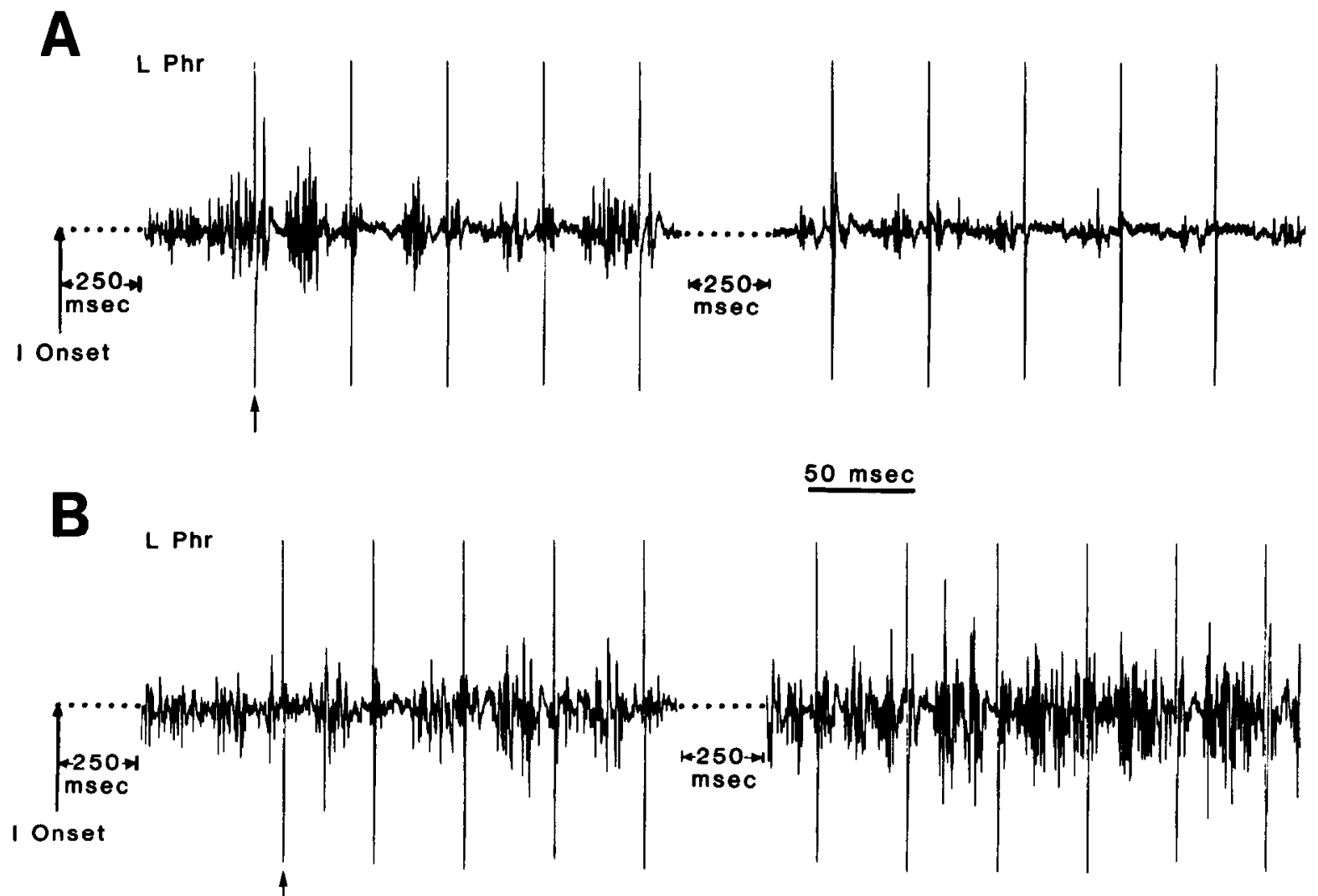

Figure 4. Short latency responses of left phrenic ( $L P h r)$ nerve to microstimulation in right VRG during inspiration. The responses in $A$ and $B$ were observed at similar stimulation parameters at two different locations in the right VRG. Control (i.e., no stimulation) inspiratory durations were similar. At both stimulation sites, short latency phrenic inhibition was observed after the very first stimulus pulse which was delivered approximately $250 \mathrm{msec}$ after inspiratory onset. The first pulse is marked by the arrow. The dots in the traces connect the beginning and end of the same inspiration and represent a 250 -msec break. $A$, When the inspiratory duration was shortened by microstimulation, the duration of phrenic inhibition tended to increase until inspiratory activity was abolished completely. $B$, However, when inspiratory duration was unaltered by microstimulation, the duration of phrenic inhibition lessened as the inspiratory effort progressed.

A

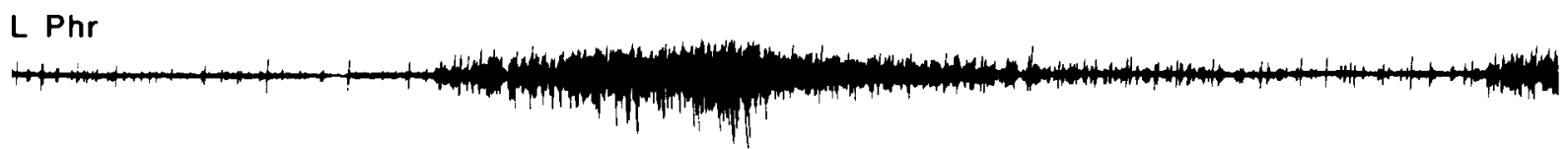

L Phr

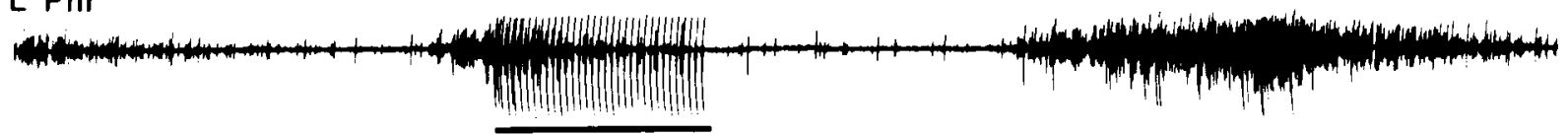

B

$1 \mathrm{sec}$

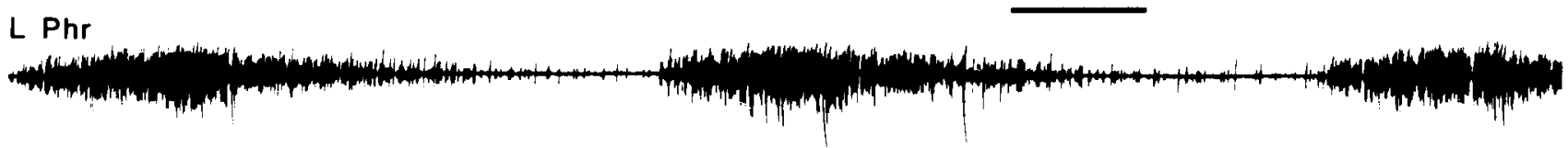

L Phr

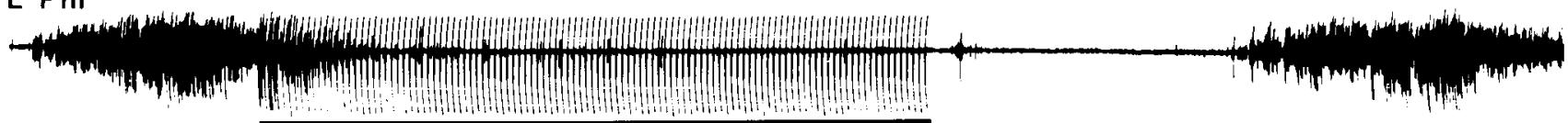

Figure 5. Effects of microstimulation in VRG on respiratory phase transitions. Low frequency $(8 \mathrm{Ho} 20 \mathrm{~Hz})$, low current (5 to 20 $\mu \mathrm{A})$ trains of pulses produced phase shifting of phrenic nerve discharge. The upper traces in $A$ and $B$ are control phrenic activity demonstrating normal durations of inspiration and expiration. The lower traces show premature termination of inspiration $(A)$ and prolongation of expiration $(B)$ elicited by microstimulation at $12 \mathrm{~Hz}$ and $20 \mu \mathrm{A}$. Similar responses were seen when stimulating at numerous and varied discrete loci in the DRG or VRG. 

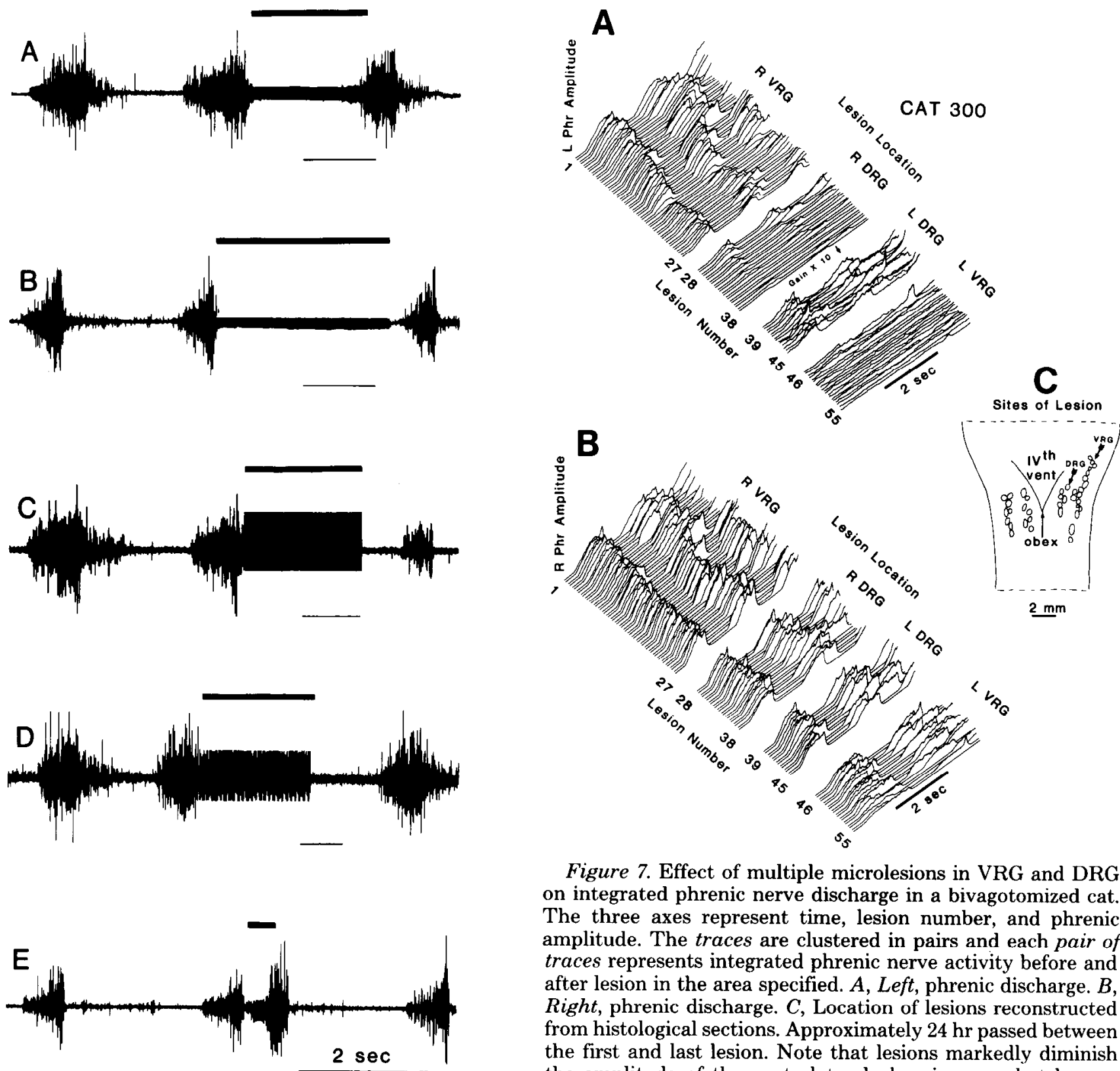

Figure 6. Effects of microstimulation in VRG during expiration. $A$, Expiratory duration was not affected by microstimulation in $64 \%$ of the stimulation trials. However, in $25 \%$ of the trials, microstimulation caused an increase in the duration of expiration. $B$, This prolongation of expiration sometimes continued only as long as the stimulus train; sometimes it continued for several seconds after the cessation of stimulation $(C) . C$, The next inspiratory effort was either reduced in amplitude or $(D)$ unaltered. $E$, In $11 \%$ of the stimulation trials, stimulation during expiration shortened that phase. Traces $A$ to $E$ were obtained during different stimulation trials in the same cat. Similar responses were observed when stimulating in the DRG. The heavy bars denote the duration of stimulus trains. The thin bars indicate $2 \mathrm{sec}$ in each panel.

successive lesions clearly established the downward trend (Fig. 7). When much larger regions of respiration-modulated activity were destroyed by passing current between pairs of adjacent microelectrodes, there was a more noticeable effect on phrenic amplitude (Fig. 8). In 2 experiments with spontaneous respiration-modulated discharge in RRI, extensive lesioning of the right VRG

Figure 7. Effect of multiple microlesions in VRG and DRG on integrated phrenic nerve discharge in a bivagotomized cat. The three axes represent time, lesion number, and phrenic amplitude. The traces are clustered in pairs and each pair of traces represents integrated phrenic nerve activity before and after lesion in the area specified. $A$, Left, phrenic discharge. $B$, Right, phrenic discharge. $C$, Location of lesions reconstructed from histological sections. Approximately $24 \mathrm{hr}$ passed between the first and last lesion. Note that lesions markedly diminish the amplitude of the contralateral phrenic nerve but have a modest effect on respiratory rhythm (see Fig. 10).

abolished the respiration-modulated activity in the RRL (Fig. 9). Despite the absence of spontaneous phasic RRL activity, microstimulation in unlesioned VRG sites near the obex still elicited a normal excitatory response in the RRL. The ipsilateral phrenic nerve appeared to be only slightly reduced in amplitude. Lesioning of the DRG also caused a reduction in the amplitude of the contralateral phrenic nerve, but this reduction was less marked than that observed with VRG microlesions.

Six animals were lesioned extensively throughout the VRG and DRG bilaterally. In 4 of the 6, the rhythm of the phrenic nerve discharge did not change abruptly over the 10 to $25 \mathrm{hr}$ required to complete the microlesions, although there were some long term drifts in respiratory rhythm and the cycle-to-cycle variance in respiratory period increased as the experiment progressed (Figs. 7, 8, 10 , and 11). The drifts in respiratory period (which often are seen in nonlesioned, paralyzed, artificially ventilated 


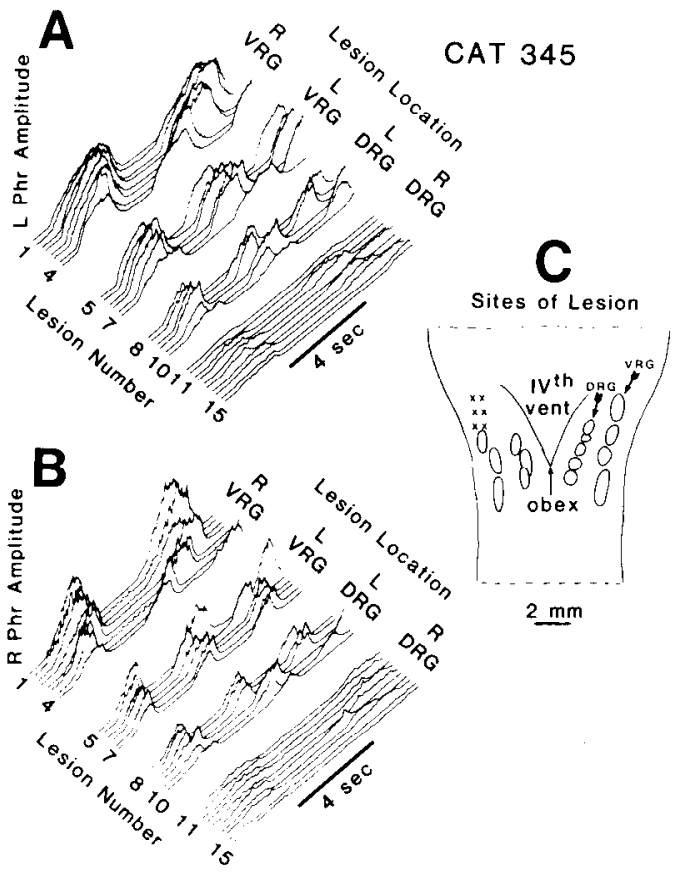

Figure 8. Effect of multiple microlesions produced by arrays of four microelectrodes in VRG and DRG on integrated phrenic nerve discharge in a single experiment. Labels and axes are as in Figure 7. $A, L e f t$, phrenic discharge. $B$, Right, phrenic discharge. $C$, Location of lesions reconstructed from histological sections. Lesions were not made in rostral portions of the left VRG because no respiration-modulated activity was found at the sites indicated by $\times \mathrm{s}$. Microlesions were made by passing current between two microelectrodes positioned approximately $1 \mathrm{~mm}$ apart in the same population. Lesions were completed in less than $10 \mathrm{hr}$ with results similar to those seen in Figure 7.

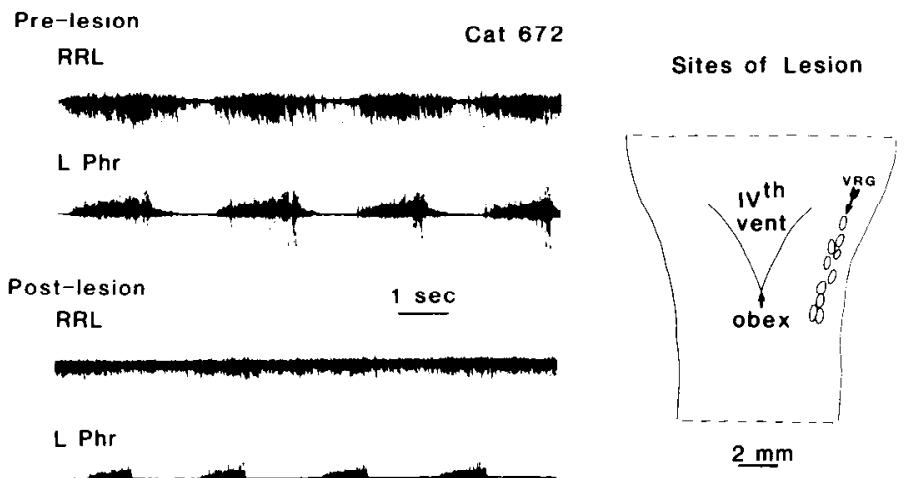

Figure 9. Effect of multiple microlesions in VRG on the activity in RRL and the left phrenic nerve ( $L \mathrm{Phr}$ ). The top two traces demonstrate prelesion control activity in the $\mathrm{L} \mathrm{Phr}$ and RRL nerves. After lesion placement at the sites indicated (lesion sites reconstructed from histological sections), $\mathrm{L} \mathrm{Phr}$ activity shows little change in the period measured, but the respiration-modulated activity of the RRI, is abolished. However, short latency responses to microstimulation (Fig. 2B) were still produced by microstimulation in the VRG at the last lesion site.

cats over similar time periods) presumably stem from slight changes in the level of anesthesia, core body temperature (controlled at $38 \pm 0.5^{\circ} \mathrm{C}$ ), metabolic state, $\mathrm{PCO}_{2}$ (end expiratory $\mathrm{CO}_{2}$ maintained at approximately
$5.0 \%$ ), etc. In the latter stages of one experiment (cat 300 , Figs. 7 and 10), respiratory frequency tended to decrease as the animal preparation began to deteriorate approximately $24 \mathrm{hr}$ after the initial anesthetization. During this time, as arterial pressure fell, the duration of both inspiration and expiration increased and became more variable. These increases occurred both between lesions (when no experimental perturbations were performed) and immediately after lesions. In 2 of the 6 cats which were lesioned extensively, the respiratory pattern became slightly apneustic as the last population (left DRG) was lesioned. However, it is important to note that the mean arterial pressure in both of these vagotomized cats fell to below $70 \mathrm{~mm} \mathrm{Hg}$ before apneusis occurred. At such low systolic pressures, apneusis is observed occasionally in vagotomized cats which have no experimental brainstem * lesions.

\section{Discussion}

The most important results of these experiments are that: $(a)$ there is a stereotyped global response to microstimulation in the DRG and VRG. This response involves a decrease in phrenic nerve activity lasting 4 to $25 \mathrm{msec}$ which occurs at a latency of 4 to 9 msec. This absence of phrenic discharge is accompanied by an excitation of the RRL nerve which has a similar onset latency and duration. (b) Extensive destruction of the DRG and VRG bilaterally has only modest effects on rhythm while significantly reducing or abolishing respiratory outflow in phrenic and recurrent laryngeal nerves.

Microstimulation. The short latency excitatory response to VRG stimulation observed in the RRL possibly is due to excitation of the cranial motoneurons known to be situated in the VRG (Bianchi, 1971; Merrill, 1970). These neurons are primarily inspiration-modulated in the VRG rostral to the obex and expiration-modulated in the caudal VRG. Both inspiratory and expiratory neurons from the right VRG project to the RRL, and this nerve typically exhibits both inspiration- and expirationmodulated activity. Based on the length of the RRL and the conduction velocities of its fibers, the response recorded on a nerve electrode to direct excitation of cranial RRL motoneurons should have a latency of 1 to $3 \mathrm{msec}$; we observed a 2- to 3-msec latency of antidromic activation of VRG inspiratory motoneurons by RRL stimulation. A direct excitation of RRL motoneurons can explain only the shortest latencies observed ( 1 to $3 \mathrm{msec}$ ) and not the entire range ( 2 to $5 \mathrm{msec}$ ). Four observations suggest that the RRL short latency excitation is due primarily to a synaptic activation of expiration-modulated neurons in the caudal VRG. (a) The RRL excitation often was "gated" in such a fashion that it was strongest during expiration and often did not even appear during inspiration. The inhibition of expiratory neurons during inspiration could easily account for this. (b) After extensive lesioning of the inspiration-modulated regions of the right VRG, the RRL ceased to exhibit a respirationmodulated pattern of activity. Despite this loss of rhythm, microstimulation in unlesioned regions of the ipsilateral VRG near the obex continued to produce a "normal" short latency excitation (i.e., similar latency and amplitude). (c) The RRL response had similar char- 
CAT 300
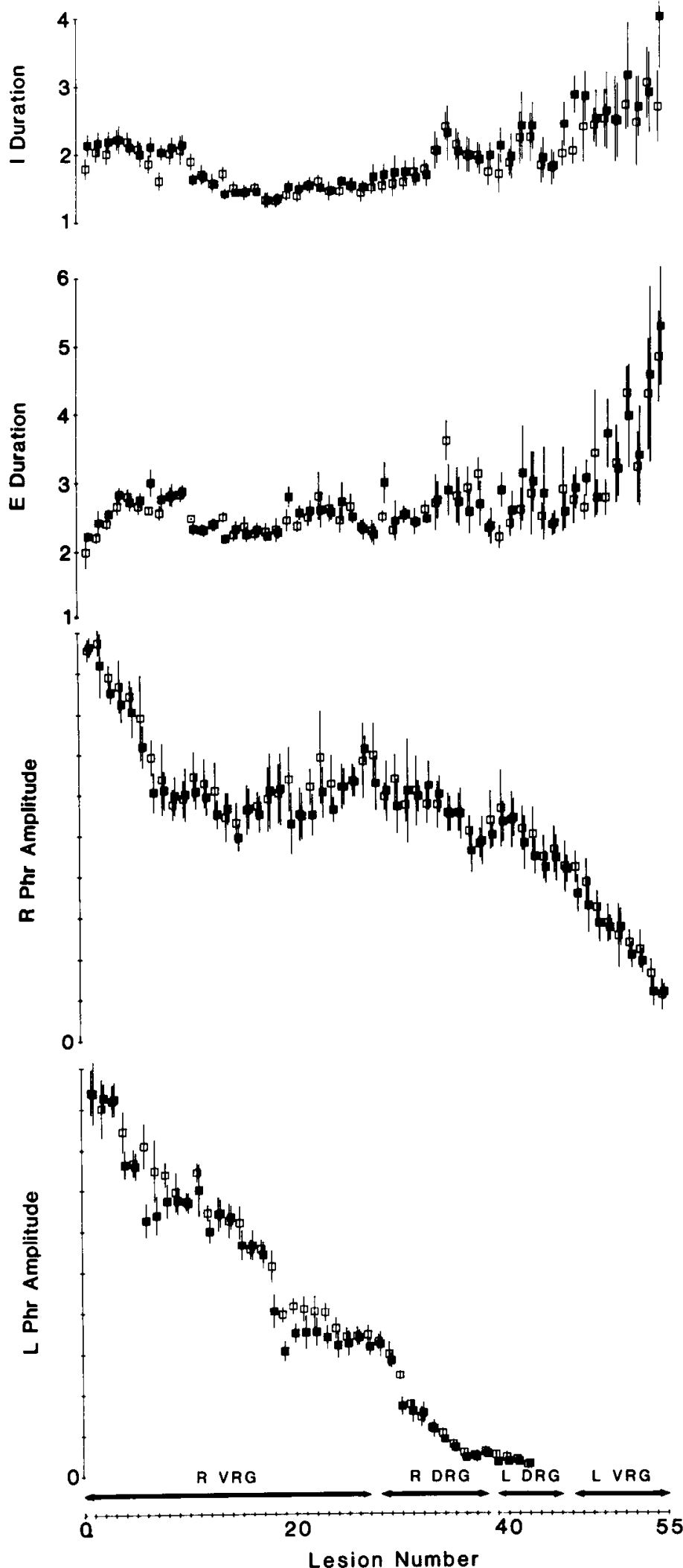

Figure 10. Inspiratory and expiratory durations (seconds) and phrenic amplitudes (arbitrary units) in cat 300 (Fig. 7) expressed as a function of lesion number. The open squares represent the mean values obtained immediately before lesion and the solid squares represent those obtained after lesion. The vertical bars represent standard deviations. Each lesion acteristics when either the VRG or DRG was stimulated. Since the response characteristics did not change when the DRG was stimulated, at least 2 to $3 \mathrm{~mm}$ away from the RRL motoneurons in VRG, it is unlikely that the response could be due to direct stimulation of VRG neurons. ( $d$ ) The latency and duration of the RRL excitation appears to be similar to that of the phrenic inhibition (see Fig. 2). It is doubtful that inspiratory neurons projecting to the phrenic nerves would be inhibited, while a select portion of inspiratory neurons which project in the RRL would be excited. Therefore, this effect was presumably due to the activation of expiration-modulated neurons. The population of expiratory neurons concentrated in the VRG caudal to the obex was not lesioned and stimulated extensively, since Bainton and Kirkwood (1978) have demonstrated that these expiratory neurons are unlikely to be essential for respiratory rhythmogenesis.

Since upper motoneurons of the phrenic nerves also are located within the VRG and DRG (Merrill, 1974; Berger, 1977), microstimulation would be expected to produce a monosynaptic excitation in those nerves. This excitation was observed only occasionally; however, in $92 \%$ of the stimulation trials, there was a period of marked inhibition of phrenic nerve discharge. The usual lack of an excitatory response in the phrenic nerve may be explained by the discreteness of the microstimulation. With the maximal monopolar stimulation current of 50 $\mu \mathrm{A}$, only those motoneurons located within 300 to $700 \mu \mathrm{m}$ of the microelectrode would be activated (Ranck, 1975). The number of phrenic upper motoneurons located within this discrete area may constitute a relatively small proportion of the total projection to the phrenic nerves.

The transient absence of phrenic activity elicited by microstimulation in the VRG and DRG could be due to an actual inhibition and/or a disfacilitation of phrenic motoneurons. Similarly, the "inhibition" of inspiratory neuronal discharge in the VRG and DRG elicited by microstimulation could be due to either an active inhibition or a disfacilitation of these neurons. The inhibition of extracellular spike discharge of these brainstem inspiratory neurons at a latency approximately 2 msec shorter than that of the phrenic inhibition suggests that phrenic motoneurons are disfacilitated.

The short latency of phrenic inhibition elicited by microstimulation throughout the VRG and DRG indicates that it involves, at most, a few synapses. Similarly, the 2- to 4-msec latency between VRG stimulation and the inhibition of extracellular spikes observed in recordings of single inspiratory units in both the VRG and DRG denotes the presence of a paucisynaptic pathway. The facts that: $(a)$ the short latency stimulation response of the phrenic nerve outflow was similar at all VRG and DRG sites, $(b)$ there was little or no mono- or disynaptic excitation of the phrenic nerve, $(c)$ the phrenic nerve inhibition had a short polysynaptic delay, $(d)$ the phrenic

did not necessarily destroy a similar number of respiratory neurons, nor did lesions occur at consistent time intervals. Approximately $24 \mathrm{hr}$ passed between the first lesion and the last. The physiological condition of this cat deteriorated seriously (mean arterial pressure, $<80 \mathrm{~mm} \mathrm{Hg}$ ) by lesion 51 . 

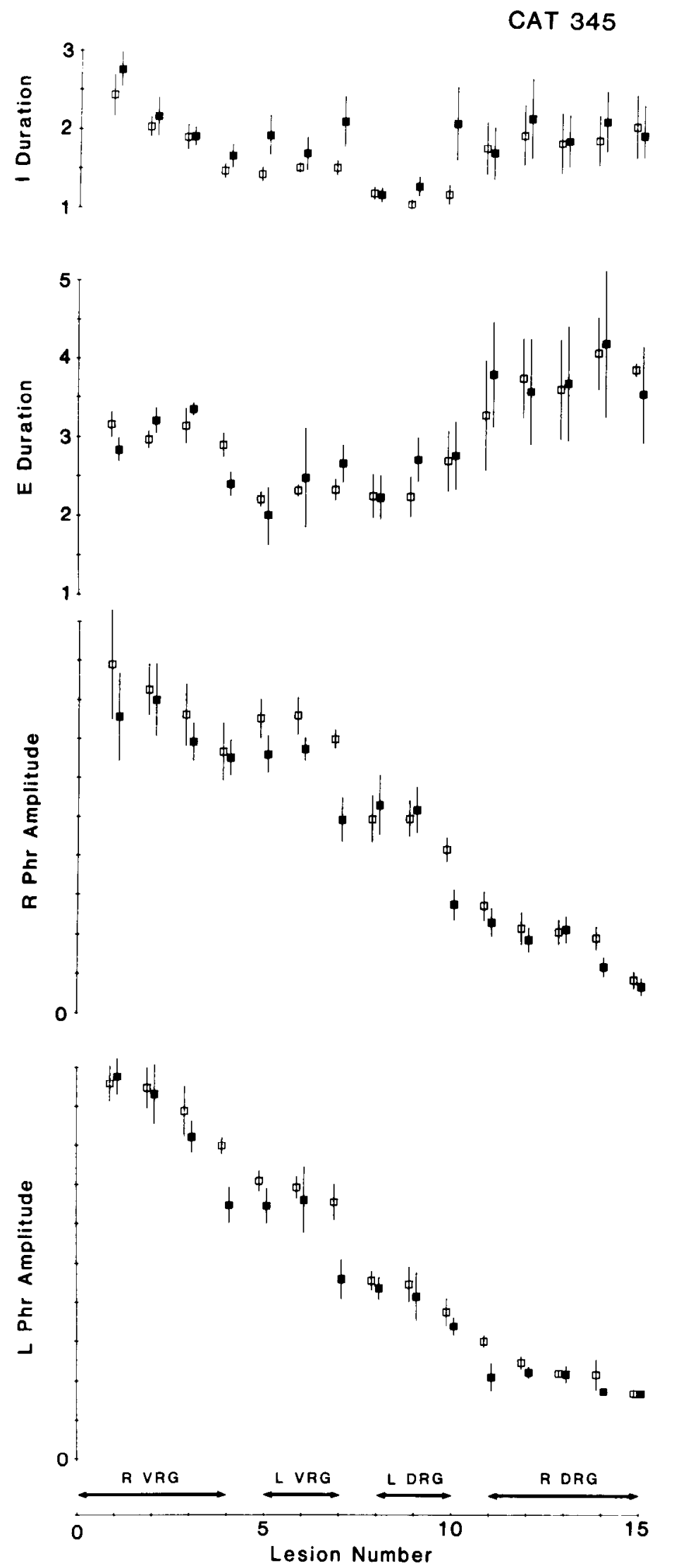

Figure 11. Inspiratory and expiratory durations (seconds) and phrenic amplitudes (arbitrary units) in cat 345 (Fig. 8). Labels and axes are as in Figure 10. Lesions were made in this cat by passing current between microelectrodes and, although still concentrated within DRG and VRG, were larger and de- nerve response saturated at modest stimulus intensities, and $(e)$ the phrenic nerve responses were similar bilaterally, suggest several possible interpretations.

(1) There is a tremendous divergence of connections between the DRG and the VRG. One candidate for this divergence would be excitatory interactions among the upper motoneurons, which could serve to ensure similar respiratory phase-locked firing patterns among respiratory neurons. This possibility is unlikely, since in this case, one would expect a significant disynaptic excitation of the phrenic nerve as neurons in the stimulated area excited a large number of distant upper motoneurons which would, in turn, excite phrenic motoneurons; this massive disynaptic excitation then could be followed by a postexcitatory inhibition. However, a disynaptic excitation at a latency of 3 to 5 msec was only rarely observed in these experiments. An important conclusion from this observation is that mutual excitatory interactions among DRG and VRG upper motoneurons are limited.

Another candidate for this divergence would be inhibitory interneurons with extensive arborization in the VRG and DRG bilaterally. Such a system of divergent inhibitory interneurons with discrete axon collaterals to various nuclei has been demonstrated in the oculomotor system (R. F. Baker, personal communication). Orthodromic activation of the inhibitory interneurons could produce the observed short latency inspiratory inhibition. Alternatively, antidromic activation of the target branches of an inhibitory neuron would produce impulses that could travel back to the cell body and excite other branches orthodromically at branch points, resulting in monosynaptic inhibition at all terminal zones. This could account for the very short latency inhibition $(<2 \mathrm{msec})$ of extracellularly recorded spikes in DRG and VRG neurons. If such inhibitory interneurons exist, it is unlikely that there are many of them and/or that they are spontaneously active during inspiration, since cross-correlation studies of spiking inspiratory neurons have not indicated the presence of significant, if any, inhibitory interactions among them (Feldman and Speck, 1981; Feldman et al., 1980; Madden and Remmers, 1981; Vachon and Duffin, 1978). These hypothetical inhibitory interneurons could be expiratory; such expiratory inhibitory neurons must exist since most DRG and VRG neurons are actively inhibited during expiration (Richter et al., 1975).

(2) The short latency phrenic response is produced by stimulation of an afferent pathway capable of producing such a response, for example, the superior laryngeal nerve afferents (Iscoe et al., 1979) or the intercostal nerve afferents (Shannon and Freeman, 1981). Stimulation of these afferents can produce similar short latency effects; however, stimulation of these afferents usually produces phase transitions, such as the prolongation of expiration and the premature termination of inspiration. Phase shifting was observed in less than half of the DRG and VRG stimulus trials. Also, since the known afferent pro-

stroyed more neural tissue. In this cat, there also was no significant change in the mean values of inspiratory and expiratory duration due to lesioning, although there was an increase in the variance. Both $\mathrm{L}$ and $\mathrm{R} \mathrm{Phr}$ amplitude decreased as lesions were made over a period of approximately $10 \mathrm{hr}$. 
jections to respiration-related regions are not as widely nor as uniformly distributed as the observed response sites (Szentagothai, 1943), we consider this possibility unlikely.

(3) There are connections from all of the regions stimulated to another population which has tremendous divergence to the brainstem respiratory nuclei. This hypothesis suggests that a population of respiration-related neurons exists outside of the DRG and VRG and has extensive and diverse connections with these populations. Such a population could receive interneurozal projections (efference copy) that are activated by microstimulation. Alternatively, its efferent axons could be activated antidromically by DRG and VRG microstimulation. Discrete, synchronous activation of this hypothetical population could produce a characteristic short latency inhibition of inspiration-related discharge as observed in the DRG, rostral VRG, and phrenic nerves and an excitation of expiration-related discharge as observed in recurrent laryngeal nerve. Depending on the activation characteristics of this population, certain patterns of stimulation also could produce shifts of the respiratory cycle.

The postinhibitory excitation observed in the phrenic nerve discharge could be due to either: $(a)$ activation of a longer latency excitatory input to the inspiratory neurons, $(b)$ a postinhibitory rebound phenomenon, or $(c)$ a pseudosynchronous discharge of inspiratory neurons which occurs after the hyperpolarizing effects of the inhibition have ended.

Unlike the ubiquitous short latency response elicited by VRG and DRG microstimulation, alterations in respiratory rhythm were observed only in less than half of the stimulation trials. The effectiveness of microstimulation in altering rhythm varied greatly in different anatomical sites; however, no correlation was found between the site of stimulation and the effect produced. In a few very sensitive locations, inspiration could be terminated by as few as three $25-\mu \mathrm{A}$ pulses delivered at $100 \mathrm{~Hz}$. Similarly, expiration could be prolonged for over $20 \mathrm{sec}$ by a $25-\mu \mathrm{A}$ pulse delivered every $100 \mathrm{msec}$. The mechanism behind this long lasting inhibition of inspiratory onset is unknown. However, since similar effects on respiratory rhythm may be elicited by stimulation of the superior laryngeal nerve (Iscoe et al., 1979) or the intercostal nerves (Shannon and Freeman, 1981), it is quite possible that VRG and DRG microstimulation may be activating these or similar pathways to produce the observed effects on respiration.

Microlesion. Successive lesions that resulted in extensive destruction of the VRG and DRG bilaterally did not alter mean respiratory rhythmicity significantly (but did increase variance) in those animals which maintained mean arterial pressures greater than 80 to $100 \mathrm{~mm} \mathrm{Hg}$. Maintenance of normal rhythm following these lesions is a very strong indication that the lesioned areas are not necessary for respiratory rhythm generation. The usual caveat about lesion experiments is appropriate to mention, i.e., loss of function following lesions does not signify that the lesioned area, including both cells and fibers of passage, generated the lost function; maintenance of function without interruption following lesions is usually a very strong indication that the lesioned area, including both cells and fibers of passage, does not play an important role in function generation. An advantage of the electrolytic microlesion technique is that it will destroy all cells and axons in an area, including cells whose activity may be missed by extracellular recording techniques (see Feldman and Cleland, 1982; Merrill, 1981). Thus, an absence of effect on rhythm following a lesion would rule out nonspiking or small extracellularly unrecordable cells in the lesioned areas as well as fibers of passage as a necessary prerequisite for rhythm generation.

The present results do not support the finding that apneusis follows from vlNTS lesions (Koepchen et al., 1974); rather, the results indicate that, if NTS lesions do not drastically insult the cardiovascular related neurons which are proximal to the region of respiratory neurons and if blood pressure remains stable, apneusis does not develop. This conclusion is supported by the observation that, in chronic cats, destruction of more than $75 \%$ of vlNTS neurons with kainic acid injections does not change the basic eupnic pattern of ventilation (Berger and Cooney, 1981). Similarly, in cardiovascular studies of the NTS, lesions of the NTS which disrupted homeostatic arterial pressure control mechanisms produced several transient changes in respiratory pattern. However, after blood pressure stabilized at hypertensive levels, respiratory patterns were not noticeably different from those observed before lesioning (Nathan and Reis, 1977). This conclusion also was made by Allen (1926) who observed that "... total destruction of the spinalmedulla and trigonum-vagi portions of the nucleus tractus solitarii produced no more permanent damage to the respiration of the guinea-pig and cat than severance of both vagus nerves."

The reduction in amplitude of the contralateral phrenic discharge following DRG and VRG microlesion is consistent with the notion that such lesions destroy upper motoneurons, since spinal projections of these neurons are known to be primarily contralateral. Despite the known brainstem interaction between the left and right VRG, the ipsilateral phrenic amplitude was not greatly affected by lesions that reduced contralateral phrenic amplitude by an order of magnitude. This suggests that the contralateral projections are not responsible for "matching" the level of respiratory outflow from the two sides of the brainstem.

There are at least four possible interpretations for the results obtained in these lesion experiments:

(1) The oscillator resides in the expiration-modulated portion of the caudal VRG. Since the expiratory neurons in the caudal VRG were not lesioned, it is possible that neurons in this region continued to produce the rhythm. The experiments of Bainton and Kirkwood (1978) strongly suggest that the caudal VRG is unlikely to be the site of rhythm generation and that the site is most likely an upper motoneuronal population. In addition, Merrill (1974) was unable to find the necessary projection from expiratory neurons in the caudal VRG to inspiratory neurons in the VRG and DRG.

(2) There is a distributed (or redundant) oscillator(s) in the DRG and/or VRG. Since every neuron in these regions was not destroyed, it is possible that the remaining neurons in these regions continued to produce the 
rhythm. However, if the oscillator was distributed throughout the DRG and rostral VRG, the system would have to be organized so that a small portion of the network could produce a rhythm of similar period to that produced by the entire network; perhaps, as the network size is decreased, there could be an increase in the cycleto-cycle variance in the respiratory pattern.

Most physical systems of coupled oscillators do change rhythm as their topology is altered. A biological example might be the coupled pacemakers located in the sinus node of the mammalian heart. Any lesion that would remove the fastest entraining oscillator would slow the rhythm. If the DRG-VRG possesses a similar arrangement, it is unlikely that the fastest oscillator(s) would have been missed in extensive randomly placed lesions in several cats. An important point to note is that the multiple microlesions made in these experiments not only destroyed most of the DRG and VRG but also served to reduce the connectivity between the small unlesioned areas which remained. 'Thus, if the oscillator is within the VRG-DRG, an important boundary condition is placed on its possible mechanisms in that small, relatively isolated fragments of the oscillator would have to have dynamics similar to the whole.

(3) There is an oscillator in the DRG and/or VRG with redundant and normally quiescent oscillators located outside of these regions. However, transient alterations in rhythm followed by "recovery" subsequent to lesions were seldon observed. It seems unlikely that a second, subordinate oscillator could assume the generation of respiratory rhythm without demonstrating observable transient effects on period. Once again, one could expect an increase in variance of the pattern if this were true.

(4) The oscillator lies outside of the DRG and VRG.

The combined results of the microstimulation and microlesion portions of this study suggest that a region (or regions) outside of the VRG and DRG might be important in the generation of respiratory pattern and that the VRG and DRG are important in determining the depth of inspiration; their role in generating respiratory rhythm needs to be critically re-examined.

\section{References}

Allen, W. F. (1926) Experimental anatomical studies on the visceral bulbospinal pathway in the cat and the guinea pig. $J$. Comp. Neurol. 42: 393-456.

Aoki, M., S. Mori, K. Kawahara, H. Watanabe, and N. Ebata (1980) Generation of spontaneous respiratory rhythm in high spinal cats. Brain Res. 202: 51-63.

Asanuma, H. (1979) Microstimulation technique. In Electrophysiological Techniques, pp. 67-77, Society for Neuroscience, Bethesda, MD.

Bainton, C. R., and P. Kirkwood (1978) The effect of carbon dioxide on the tonic and the rhythmic discharges of expiratory bulbospinal neurones. J. Physiol. (Lond.) 296: 291-314.

Berger, A. J. (1977) Dorsal respiratory group neurons in the medulla of the cat: Spinal projections, responses to lung inflation and superior laryngeal nerve stimulation. Brain Res. 113: 231-254.

Berger, A. J., and K. A. Cooney (1981) Hypoxic and hypercapnic ventilatory drives following kainic acid (KA) lesions of the ventrolateral nucleus of the solitary tract (vINTS). Fed. Proc. 40: 429 .
Bianchi, A. L. (1971) Localisation et etude des neurones respiratoires bulbaires. J. Physiol. (Paris) 36: 5-40.

Binet, L. M., V. Strumza, and J. M. Strumza-Poutonnet (1953) Sur la 'respiration' experimentalle medullaire. J. Physiol. (Paris) 45: 41-43.

Brodie, D. A., and H. L. Borison (1957) Evidence for a medullary inspiratory pacemaker: Functional concept of central regulation of respiration. Am. J. Physiol. 188: 347-354.

Bystrzycka, E. K. (1980) Afferent projections to the dorsal and ventral respiratory nuclei in the medulla oblongata of the cat studied by the horseradish peroxidase technique. Brain Res. 185: 59-66.

Cohen, M. I. (1968) Discharge patterns of brain-stem respiratory neurons in relation to carbon dioxide tension. J. Neurophysiol. 31: 142-165.

Cohen, M. I. (1979) Neurogenesis of respiratory rhythm in the mammal. Physiol. Rev. 59: 1105-1173.

Feldman, J. L., and C. L. Cleland (1982) Possible roles of pacemaker neurons in mammalian respiratory rhythmogenesis. In Cellular Pacemakers, D. O. Carpenter, ed., Vol. II, pp. 104-128, John Wiley and Sons, New York.

Feldman, J. L., and D. F. Speck (1981) Short time-scale correlated activity among medullary inspiratory neurons. Fed. Proc. 40: 429.

Feldman, J. L., M. I. Cohen, and P. Wolotsky (1976) Powerful inhibition of pontine respiratory neurons by pulmonary afferent activity. Brain Res, 104: 341-346.

Feldman, J. L., D. Sommer, and M. I. Cohen (1980) Short time scale correlations between discharges of medullary respiratory neurons. J. Neurophysiol. 43: 1284-1295.

Gromysz, H., and W. A. Karczewski (1980) Generation of respiratory pattern in the rabbit-brainstem transections revisited. Acta Neurobiol. Exp. (Warsz.) 40: 985-992.

Gromysz, H., W. A. Karczewski, E. Naslonska, K. Ruszczyk, and K. Sroczynska (1980) Effects of reversible elimination of some bulbar structures on the generation of respiratory pattern in rabbits. Acta Neurobiol. Exp. (Warsz.) 40: 507-514.

Hoff, H. E., and C. G. Breckenridge (1949) The medullary origin of respiratory periodicity in the dog. Am. J. Physiol. 158: 157-172.

Hukahara, T., Jr. (1976) Functional organization of brain stem respiratory neurons and its afferences. In Respiratory Centres and Afferent Systems, B. Duron, ed., pp. 41-53, Institut National de la Santé et de la Recherche Médicale, Paris.

Iscoe, S., J. L. Feldman, and M. I. Cohen (1979) Properties of inspiratory termination by superior laryngeal and vagal stimulation. Respir. Physiol. 36: 353-366.

Kalia, M., J. L. Feldman, and M. I. Cohen (1979) Afferent projections to inspiratory neuronal region of the ventrolateral nucleus of the tractus solitarius in the cat. Brain Res. 171: 135-141.

Kurczewski, W. A., and H. Gromysz (1980) The "split respiratory centre," lessons from brainstem transections. Adv. Physiol. Sci. 10: 587-594.

Koepchen, H. P., H. Lazar, and J. Borchert (1974) On the role of the nucleus infrasolitarius in the determination of respiratory periodicity. Proc. Intl. Union Physiol. Sci. 11: 81.

Madden, K. P., and J. E. Remmers (1981) Short time-scale correlations of the spike activity of neighboring respiratory neurons within the nucleus tractus solitarius. Fed. Proc. 40: 427.

Merrill, E. G. (1970) The lateral respiratory neurons of the medulla, their associations with nucleus ambiguus, nucleus retroambigualis, the spinal accessory nucleus and the spinal cord. Brain Res. 24: 11-28.

Merrill, E. G. (1974) Finding a respiratory function for the medullary respiratory neurons. In Essays on the Nervous System, R. Bellairs and E. G. Gray, eds., pp. 451-486, Clarendon, Oxford. 
Merrill, E. G. (1981) Where are the real respiratory neurons? Fed. Proc. 40: 2389-2394.

Mitchell, R. A., and A. J. Berger (1981) Neural regulation of respiration. In Regulation of Breathing, T. F. Hornbein, ed., pp. 541-620, Marcel Dekker, Inc., New York.

Nathan, M. A., and D. J. Reis (1977) Chronic labile hypertension produced by lesions of nucleus tractus solitarius in the cat. Circ. Res. 40: 72-81.

Oberholzer, J. H., and W. O. Tofani (1965) The neural control of respiration. In Handbook of Physiology. Vol. II: Neurophysiology, pp. 1111-1129, The American Physiological Society, Washington, DC.

Pitts, R. F., H. W. Magoun, and S. W. Ranson (1939) The origin of respiratory rhythmicity. Am. J. Physiol. 127: 654-670.

Ranck, J. B., Jr. (1975) Which elements are excited in electrical stimulation of mammalian central nervous system: A review. Brain Res. 98: 417-440.

Richter, D. W., F. Heyde, and M. Gabriel (1975) Intracellular recordings from different types of medullary respiratory neurons of the cat. J. Neurophysiol. 38: 1162-1171.

Shannon, R., and D. Freeman (1981) Nucleus retroambigualis respiratory neurons: Responses to intercostal and abdominal muscle afferents. Respir. Physiol. 45: 357-375.

St. John, W. M., and S. C. Wang (1977a) Alteration from apncusis to more regular rhythmic respiration in decerebrate cats. Respir. Physiol. 31: 91-106.

St. John, W. M., and S. C. Wang (1977b) Response of medullary respiratory neurons to hypercapnia and isocapnic hypoxia. J. Appl. Physiol. 43: 812-821.

St. John, W. J., D. Bartlett, Jr., K. V. Knuth, and J. -C. Hwang (1981) Brain stem genesis of automatic ventilatory patterns independent of spinal mechanisms. J. Appl. Physiol. 51: 204210.

Szentagothai, J. (1943) Die lokalisation dir kehlkopfmuskulatur in den vaguskerernen. Z. Anat. Entwicklungsgesch. 112: 704710.

Vachon, B. R., and J. Duffin (1978) Cross-correlation of medullary respiratory neurons in the cat. Exp. Neurol. 61: 15-30.

Vibert, J. F., F. Bertrand, M. Denavit-Saubie, and A. Hugelin (1976) Three dimensional representation of bulbo-pontine respiratory networks architecture from unit density maps. Brain Res. 114: 227-244.

Wang, S. C., S. H. Ngai, and M. J. Frumin (1957) Organization of central respiratory mechanisms in the brain stem of the cat: Genesis of normal respiratory rhythmicity. Am. J. Physiol. 190: 333-342.

Wyman, R. J. (1977) Neural generation of the breathing rhythm. Annu. Rev. Physiol. 39: 417-448. 